\title{
Serum concentrations of Flt-3 ligand in rheumatic diseases
}

\author{
Kayo Nakamura*, Noriko Nakatsuka*, Masatoshi Jinnin**, Takamitsu Makino, Ikko Kajihara, \\ Katsunari Makino, Noritoshi Honda, Kuniko Inoue, Satoshi Fukushima, Hironobu Ihn
}

Department of Dermatology and Plastic Surgery, Faculty of Life Sciences, Kumamoto University, Kumamoto, Japan.

\begin{abstract}
Summary Fms-like tyrosine kinase 3 (Flt-3) is a cytokine receptor expressed on the surface of bonemarrow progenitor of hematopoietic cells. Flt-3 ligands are produced by peripheral blood mononuclear cells, and found in various human body fluids. Flt-3 signal is involved in the regulation of vessel formation as well as $B$ cell differentiation, suggesting that Flt-3 signal contributes to the pathogenesis of vascular abnormalities and immune dysregulation in rheumatic diseases. The aim of the present study is to examine serum Flt-3 ligand levels in patients with various rheumatic diseases, and to evaluate the possibility that serum Flt-3 ligand levels can be a useful disease marker. Sera were obtained from 20 dermatomyositis (DM) patients, 36 systemic sclerosis (SSc) patients, 10 systemic lupus erythematosus (SLE) patients, 10 scleroderma spectrum disorder (SSD) patients, 4 mixed connective tissue disease (MCTD) patients, and 12 normal subjects. Flt-3 ligand levels were determined with ELISA. Serum Flt-3 ligand levels were significantly elevated in patients with DM, SSc, SSD and MCTD compared to those in normal subjects. DM patients with elevated Flt-3 ligand levels were accompanied with significantly increased CRP levels and increased frequency of heliotrope rash than those with normal levels. In addition, SSc patients with elevated Flt-3 ligand levels showed significantly reduced frequency of nailfold bleeding. Serum Flt-3 ligand levels can be a marker of cutaneous manifestation in DM and a marker of microangiopathy in SSc. Clarifying the role of Flt-3 ligand in rheumatic diseases may lead to further understanding of these diseases and new therapeutic approaches.
\end{abstract}

Keywords: Flt-3, dermatomyositis, systemic sclerosis, systemic lupus erythematosus

\section{Introduction}

Immune dysfunction and vascular abnormalities are thought to be the common features in rheumatic diseases including rheumatoid arthritis (RA), polymyositis/dermatomyositis (PM/DM), systemic sclerosis (SSc), systemic lupus erythematosus (SLE), mixed connective tissue disease (MCTD) or Sjögren's syndrome $(\mathrm{SjS})(1,2)$. For example, autoantibodies, Raynaud's phenomenon, nailfold bleeding (NFB) and skin ulcers are frequently seen in various rheumatic diseases. These vascular abnormalities are characterized by uncontrolled regeneration of the vasculature and

\footnotetext{
*These authors contributed equally to this works.

**Address correspondence to:

Dr. Masatoshi Jinnin, Department of Dermatology and Plastic Surgery, Faculty of Life Sciences, Kumamoto University, 1-1-

1 Honjo, Kumamoto 860-8556, Japan.

E-mail: mjin@kumamoto-u.ac.jp
}

vascular losses due to defective maintenance of the vasculature (3). However, the mechanism is hardly understood, and needs to be clarified.

Fms-like tyrosine kinase 3 (Flt-3), also known as $\mathrm{CD} 135$, is a cytokine receptor expressed on the surface of bone marrow-derived hematopoietic progenitor cells. Activation of the receptor has been reported to regulate vessel formation as well as the blood cell differentiation. For example, Flt-3 signal is thought to play roles in the angiogenesis of malignant tumors, the process of $\mathrm{B}$ cell differentiation, immune response to virus infection, and aging (4-7). Furthermore, Flt-3 mutations are found in patients with leukemia (8). Thus, there is a possibility that Flt-3 signal is involved in the pathogenesis of vascular abnormalities and immune dysregulation in rheumatic diseases as well as similar dysregulation of immunity and angiogenesis such as malignancy, infection and aging.

On the other hand, Flt-3 ligands, approximately $30 \mathrm{kDa}$ transmembrane glycoproteins, are produced 
by peripheral blood mononuclear cells (9), and can be found as soluble homodimeric proteins in various human body fluids including serum. So far, several previous studies have suggested the significance of Flt3 ligand levels in autoimmune arthritis such as RA and primary Sjogren's syndrome: there was a significant correlation between serum Flt-3 ligand levels and disease activity in RA patients $(10,11)$. However, the expression of Flt-3 ligand in other rheumatic diseases has yet to be elucidated. Therefore, in this study, we examined serum Flt-3 ligand levels in patients with various rheumatic diseases, and tried to evaluate the possibility that serum levels of Flt-3 ligand can be a useful disease marker.

\section{Materials and Methods}

\subsection{Clinical assessment and patient material}

Patients with SSc and SLE fulfilled the criteria proposed by the American College of Rheumatology (ACR) $(12,13)$. All SSc patients were grouped into diffuse cutaneous SSc $(\mathrm{dcSSc})$ and limited cutaneous SSc (lcSSc) according to the classification system proposed by LeRoy et al. (14). The concept of scleroderma spectrum disorder (SSD) was originally proposed by Maricq et al. to unify typical SSc, early forms of SSc and closely related disorders $(15,16)$. After that, Ihn et al. redefined SSD as patients that did not fulfill the ACR criteria of SSc but were thought to develop SSc in the future, and proposed a new diagnostic method (17). Classical DM patients were diagnosed based on the criteria proposed by Bohan and Peter $(18,19)$. Patients with clinically and histopathologically typical cutaneous lesions but without myositis were diagnosed as clinically amyopathic DM (CADM) according to the previous criteria (20). MCTD patients had clinical features of SLE, SSc and PM/DM and anti-U1RNP antibodies, but did not satisfy the criteria for these connective tissue diseases $(12,13,18,19)$, and were diagnosed according to the criteria of Alarcón-Segovia $(21,22)$.

Sera were obtained from randomly chosen 15 classical DM patients (age range: 27-86 years, mean \pm SD: $54.1 \pm 18.4$; disease duration range: 1-24 months, mean $\pm \mathrm{SD}: 7.4 \pm 7.5$ ), $5 \mathrm{CADM}$ patients (age range: 33-81 years, mean \pm SD: $61.4 \pm 19.4$; disease duration range: 1-108 months, mean $\pm \mathrm{SD}: 31.2 \pm 38.5)$, 36 patients with SSc (13 dcSSc and $23 \mathrm{lcSSc}$, age range: 24-85 years, mean \pm SD: $62.2 \pm 14.6$; disease duration range: $1-768$ months, mean $\pm \mathrm{SD}: 75.0 \pm 151.7), 10$ SSD patients (age range: $44-75$ years, mean \pm SD: 54.9 \pm 10.7 ; disease duration range: $1-120$ months, mean \pm SD: $27.9 \pm 40.1$ ), 10 SLE patients (age range: $23-$ 57 years, mean \pm SD: $35.0 \pm 11.9$; disease duration range: $2-84$ months, mean $\pm \mathrm{SD}: 21.3 \pm 27.6)$ and 4 MCTD patients (age range: $44-73$ years, mean \pm SD:
$59.8 \pm 12.6$; disease duration range: $24-228$ months, mean \pm SD: $123.0 \pm 83.4)$. Clinical and laboratory data reported in this study were obtained at the time of serum sampling. None of the patients received systemic treatments such as steroid or immunosuppressant at the serum sampling.

Control serum samples were also collected from 12 normal subjects (age range $=32-81$ years, mean $\pm \mathrm{SD}$ : $47.1 \pm 16.4$ ). All serum samples were stored at $-80^{\circ} \mathrm{C}$ prior to use. Institutional review board approval and written informed consent were obtained before patients and healthy volunteers were entered into this study according to the Declaration of Helsinki.

\subsection{Nailfold bleeding}

Nailfold bleeding (NFB) was evaluated macroscopically by the presence of splinter hemorrhages of the nailfold capillaries in more than one finger by 2 investigators in a blinded manner.

\subsection{Serum concentrations of Flt-3 ligand}

Levels of serum Flt-3 ligand were measured with a specific ELISA kit (R\&D Systems, Minneapolis, MN, USA) (23). Briefly, anti-Flt-3 ligand monoclonal antibodies were precoated onto microtiter wells. Aliquots of serum were added to each well, followed by the addition of peroxidase-conjugated antibodies to Flt-3 ligand. Color was developed with hydrogen peroxide and tetramethylbenzidine peroxidase, and the absorbance at $450 \mathrm{~nm}$ was measured. Wavelength correction was performed by absorbance at $540 \mathrm{~nm}$. The concentration of Flt-3 ligand in each sample was determined by interpolation from a standard curve.

\subsection{Statistical analysis}

Statistical analysis was carried out with Mann-Whitney $\mathrm{U}$ test for the comparison of medians, and Fisher's exact probability test for the analysis of frequency. $p$ values less than 0.05 were considered significant. Receiver operating characteristic (ROC) curve analysis was performed to evaluate the diagnostic performance of Flt-3 ligand levels using GraphPad Prism ver. 5.01 (MDF software, Tokyo, Japan).

\section{Results}

\subsection{Serum concentrations of Flt-3 ligand in patients with various rheumatic diseases}

The serum Flt-3 ligand levels in patients with various rheumatic diseases are shown in Figure 1. Serum samples were obtained from $20 \mathrm{DM}$ patients (including 5 CADM patients), 36 SSc patients, 10 SSD patients, 10 SLE patients, 4 MCTD patients, and 12 normal 
subjects. SSD patients did not fulfill the criteria of SSc but were thought to develop SSc in the future, while CADM patients had typical cutaneous lesions of DM but lacked myositis $(15-17,20)$.

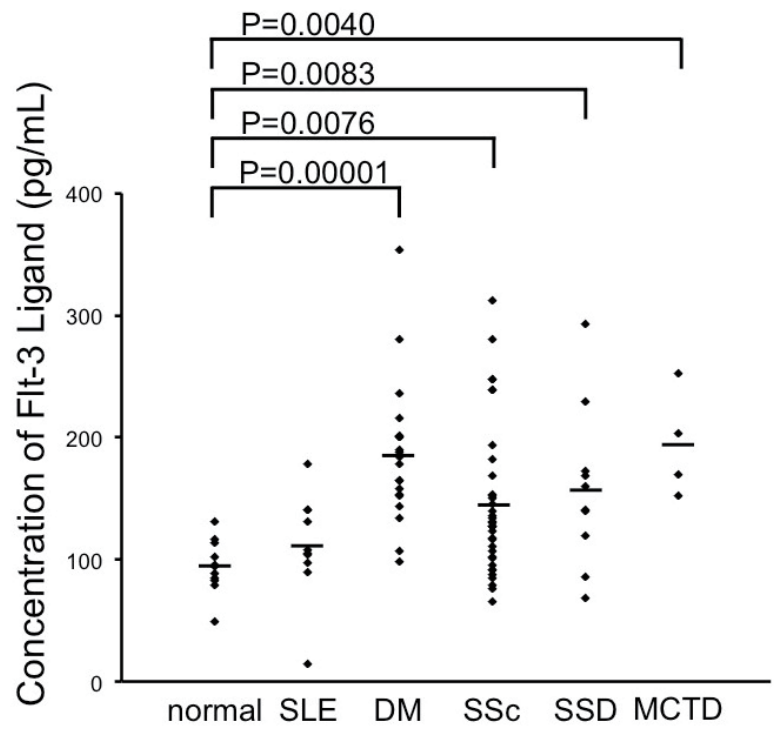

Figure 1. The serum levels of Flt-3 ligand in rheumatic diseases. Levels of Flt-3 ligand in sera of patients with systemic lupus erythematosus (SLE, $n=10$ ), dermatomyositis (DM, $n=20$ ), systemic sclerosis (SSc, $n=36$ ), scleroderma spectrum disorder (SSD, $\mathrm{n}=10$ ), mixed connective tissue disease (MCTD, $n=4)$ and normal subjects $(n=12)$ were analyzed with ELISA. Bars show the means.
The mean age of normal subjects was 47.1 years, and the ratio of female was $75.0 \%$. Summary of clinical/laboratory features in patients with these rheumatic diseases enrolled in this study are shown in Tables and Supplemental Tables. For example, in DM patients, the mean age at the serum sampling was 56.0 years, and mean CK levels were 2,400.8 IU/L (normal values; 57-284 IU/L for male and 45-176 IU/L for female) (Table 1). Among the rheumatic diseases, DM patients had elevated serum Flt-3 ligand concentration (mean \pm SD: $185.1 \pm 57.4 \mathrm{pg} / \mathrm{mL}$ ), and there was a statistically significant difference in the values between DM patients and normal subjects $(94.4 \pm 21.0 \mathrm{pg} / \mathrm{mL}, p$ $=0.000011$, Figure 1). According to the manufacturer's data, mean normal serum Flt-3 ligand level was 93.9 pg/ $\mathrm{mL}$, which is consistent with our results. In ROC curve analysis for serum Flt-3 ligand levels to distinguish DM patients from normal subjects, the area under the curve (AUC) was 0.9708 (95\% confidence interval; 0.92191.020, Figure 2A): AUC more than 0.9 means that the level of Flt-3 ligand is useful for diagnosis of DM patients. When the most optical cut-off point was set at $132.8 \mathrm{pg} / \mathrm{mL}$ according to the Youden index (0.90), the sensitivity was $90.0 \%$ and the specificity was $100.0 \%$. There were no significant differences between patients with classical DM and CADM (189.9 \pm 52.4 vs. 170.8 $\pm 55.0 \mathrm{pg} / \mathrm{mL}, p=0.51$, Figure 2B).

Levels of serum Flt-3 ligand also showed significant elevation in patients with SSc compared to in normal

Table 1. Correlation of serum Flt-3 ligand levels with clinical/serological features in patients with dermatomyositis

\begin{tabular}{|c|c|c|c|}
\hline \multirow{2}{*}{ Items } & \multirow{2}{*}{ All patients $(n=20)$} & \multicolumn{2}{|c|}{ Serum Flt-3 ligand } \\
\hline & & $\begin{array}{l}\text { Patients with elevated Flt-3 } \\
\text { ligand levels }(n=10)\end{array}$ & $\begin{array}{l}\text { Patients with normal Flt-3 } \\
\text { ligand levels }(n=10)\end{array}$ \\
\hline Age at the time of serum sampling (mean years) & 56.0 & 61.9 & 50.0 \\
\hline Gender (ratio of female, \%) & 60.0 & 50.0 & 70.0 \\
\hline Duration of disease (mean months) & 13.4 & 6.3 & 20.3 \\
\hline Type (DM:CADM) & $15: 5$ & $8: 2$ & $7: 3$ \\
\hline \multicolumn{4}{|l|}{ Clinical features } \\
\hline Gottron's sign (\%) & 83.3 & 100.0 & 70.0 \\
\hline Heliotrope rash $(\%)$ & 75.0 & $100.0^{*}$ & 50.0 \\
\hline \multicolumn{4}{|l|}{ Laboratory features } \\
\hline $\operatorname{IgG}(\mathrm{mg} / \mathrm{dL})$ & 1379.2 & 1365.4 & 1392.9 \\
\hline CK (IU/L) & 2400.8 & 2155.2 & 2646.4 \\
\hline Myoglobin (ng/mL) & 694.6 & 602.6 & 786.7 \\
\hline Aldolase (U/L) & 37.0 & 21.6 & 50.7 \\
\hline $\mathrm{CRP}(\mathrm{mg} / \mathrm{dL})$ & 0.83 & $1.48^{*}$ & 0.19 \\
\hline \multicolumn{4}{|l|}{ Organ involvement } \\
\hline Muscle weakness (\%) & 76.5 & 87.5 & 66.7 \\
\hline Interstitial kung disease $(\%)$ & 35.3 & 42.6 & 30.0 \\
\hline Dysphasia (\%) & 30.8 & 40.0 & 20.0 \\
\hline Joint (\%) & 28.6 & 50.0 & 20.0 \\
\hline \multicolumn{4}{|l|}{ ANA specificity } \\
\hline ANA $(+)$ & 50.0 & 70.0 & 30.0 \\
\hline (levels, index) & 48.6 & 67.3 & 20.5 \\
\hline Anti-Jo-1 (\%) & 10.0 & 10.0 & 10.0 \\
\hline (levels, IU/mL) & 1.68 & 1.74 & 1.61 \\
\hline
\end{tabular}

The cut-off vale was set at the mean $+4 \mathrm{SD}$ of the value in healthy control subjects. DM, dermatomyositis; CADM, clinicallyamyopathic dermatomyositis; CK, creatin kinase; ANA, antinuclear antibodies; Anti-Jo-1, Anti-Jo-1 antibodies. ${ }^{*} p<0.05$, versus patients with normal Flt-3 ligand levels using Fisher's exact probability test. 
A

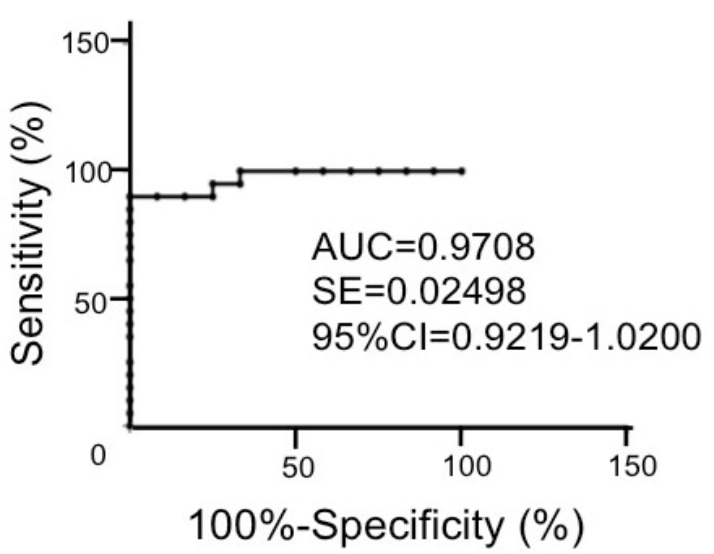

B

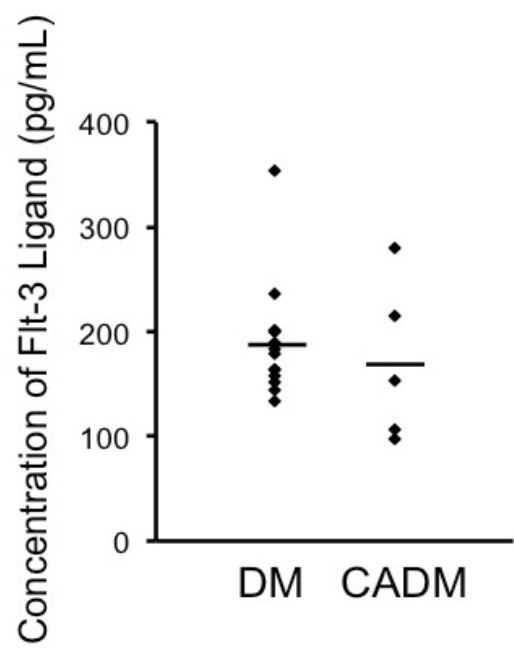

Figure 2. The serum levels of Flt-3 ligand in dermatomyositis. (A) Receiver operating characteristic (ROC) curve for serum Flt-3 ligand to distinguish dermatomyositis (DM) patients from normal subjects. AUC; areas under curves, SE; standard error, CI; confidence interval. (B) Levels of Flt3 ligand in sera of patients with classical DM $(n=15)$ and clinically amyopathic DM (CADM, $n=5)$ were analyzed with ELISA. Bars show the means.

subjects $(144.7 \pm 60.9$ vs. $94.4 \pm 21.0 \mathrm{pg} / \mathrm{mL}, p=$ 0.0026). In ROC curve analysis for serum Flt-3 ligand levels to distinguish SSc patients from normal subjects, AUC was 0.7928 (95\% confidence interval; 0.6655 0.9202 , Figure 3A): AUC more than 0.7 indicates sufficient diagnostic performance of serum Flt-3 ligand levels. When the most optical cut-off point was set at $114.8 \mathrm{pg} / \mathrm{mL}$ by the Youden index $(0.56)$, the sensitivity and specificity were $63.9 \%$ and $91.7 \%$, respectively. We could not find any significant differences in Flt-3 ligand levels between dcSSc patients and lcSSc patients $(139.8$ \pm 69.1 vs. $147.6 \pm 57.3 \mathrm{pg} / \mathrm{mL}, p=0.72$, Figure 3B).

Patients with SSD and MCTD also showed significantly higher serum Flt-3 ligand levels than normal subjects $(157.7 \pm 65.9$ vs. $94.4 \pm 21.0 \mathrm{pg} /$ $\mathrm{mL}, p=0.0083$ and $194.3 \pm 44.1$ vs. $94.4 \pm 21.0 \mathrm{pg} /$ $\mathrm{mL}, p=0.0040$, respectively) (Supplemental Tables 1 and 2, http://www.biosciencetrends.com/docindex. php? year $=2015 \&$ kanno $=5$ ). On the other hand, serum Flt-3 ligand levels were slightly higher in patients
A

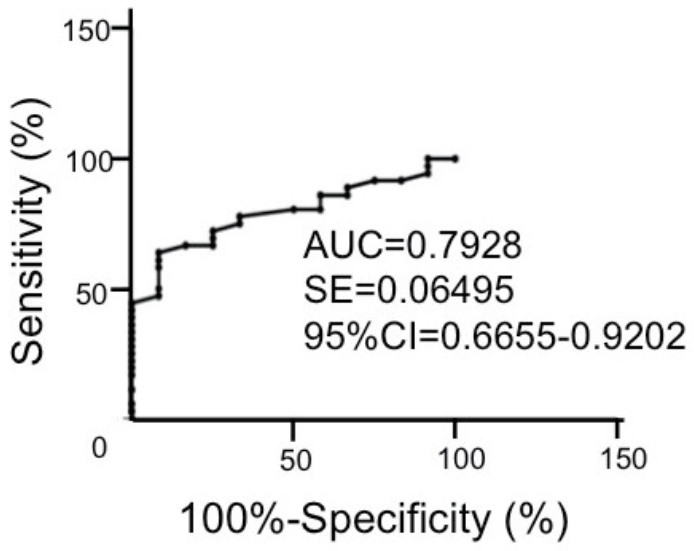

B

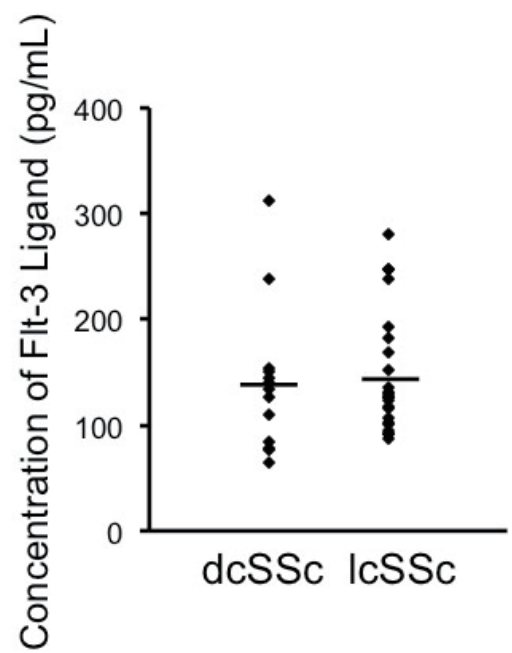

Figure 3. The serum levels of Flt-3 ligand in systemic sclerosis. (A) Receiver operating characteristic (ROC) curve for serum Flt-3 ligand to distinguish systemic sclerosis (SSc) patients from normal subjects. AUC; areas under curves, SE; standard error, CI; confidence interval. (B) Levels of Flt-3 ligand in sera of patients with diffuse cutaneous SSc (dcSSc, $n$ $=13)$ and limited cutaneous SSc $(1 \mathrm{cSSc}, n=23)$ were analyzed with ELISA. Bars show the means.

with SLE compared with normal subjects, but the difference was not statistically significant $(111.0 \pm$ 43.3 vs. $94.4 \pm 21.0 \mathrm{pg} / \mathrm{mL}, p=0.25$ ) (Supplemental Table 3, http://www.biosciencetrends.com/docindex. php? year $=2015 \&$ kanno $=5$ ).

Taken together, significant increases of serum Flt-3 ligand levels were found in patients with DM, SSc, SSD, and MCTD. When the cut-off value was set at the mean +2 SD of Flt-3 ligand levels in normal subjects (136.4 $\mathrm{pg} / \mathrm{mL}$ ), all the values in normal subjects were below the cut-off. On the other hand, increased serum Flt-3 ligand levels were found in 17 of the $20 \mathrm{DM}$ patients $(85.0 \%)$, 14 of the $36 \mathrm{SSc}$ patients $(38.9 \%), 7$ of the $10 \mathrm{SSD}$ patients $(70.0 \%)$, all of the 4 MCTD patients $(100.0 \%)$ and 3 of the 10 SLE patients (30.0\%). Furthermore, when the cut-off was set at mean $+4 \mathrm{SD}$ of values in normal subjects $(178.5 \mathrm{pg} / \mathrm{mL})$, increased serum Flt-3 ligand levels were seen in 10 of the $20 \mathrm{DM}$ patients $(50.0 \%), 8$ of the $36 \mathrm{SSc}$ patients $(22.2 \%), 2$ of the $10 \mathrm{SSD}$ patients $(20.0 \%)$, and 2 of the 4 MCTD patients $(50.0 \%)$. 
Table 2. Correlation of serum Flt-3 ligand levels with clinical/serological features in patients with systemic sclerosis

\begin{tabular}{|c|c|c|c|}
\hline \multirow{2}{*}{ Items } & \multirow{2}{*}{ All patients $(n=36)$} & \multicolumn{2}{|c|}{ Serum Flt-3 ligand } \\
\hline & & $\begin{array}{c}\text { Patients with elevated Flt-3 } \\
\text { ligand levels }(n=14)\end{array}$ & $\begin{array}{c}\text { Patients with normal Flt-3 } \\
\text { ligand levels }(n=22)\end{array}$ \\
\hline Age at the time of serum sampling (mean years) & 62.2 & 64.9 & 60.3 \\
\hline Gender (ratio of female, \%) & 77.7 & 75.0 & 77.2 \\
\hline Duration of disease (mean months) & 75.0 & 58.2 & 86.6 \\
\hline Type (diffuse:limited) & $13: 23$ & $6: 8$ & $7: 15$ \\
\hline m-TSS & 11.0 & 11.6 & 10.5 \\
\hline \multicolumn{4}{|l|}{ Clinical features } \\
\hline Pitting scars (\%) & 43.8 & 50.0 & 40.0 \\
\hline Ulcers $(\%)$ & 32.0 & 20.0 & 40.0 \\
\hline Nailfold bleeding (\%) & 48.7 & $26.7^{*}$ & 63.6 \\
\hline Raynaud's phenomenon (\%) & 90.3 & 100.0 & 84.2 \\
\hline Telangiectasia (\%) & 23.1 & 18.2 & 26.7 \\
\hline Contracture of phalanges (\%) & 85.7 & 100.0 & 77.8 \\
\hline Calcinosis $(\%)$ & 5.9 & 0.0 & 10.0 \\
\hline Diffuse pigmentation (\%) & 28.6 & 42.9 & 21.4 \\
\hline Short SF (\%) & 75.0 & 85.7 & 70.6 \\
\hline Sicca symptoms $(\%)$ & 53.9 & 45.5 & 60.0 \\
\hline \multicolumn{4}{|l|}{ Laboratory features } \\
\hline $\mathrm{CRP}(\mathrm{mg} / \mathrm{dL})$ & 0.75 & 0.95 & 0.57 \\
\hline \multicolumn{4}{|l|}{ Organ involvement } \\
\hline Pulmonary fibrosis (\%) & 37.1 & 35.7 & 38.1 \\
\hline Mean \%VC (\%) & 102.9 & 100.0 & 104.9 \\
\hline Mean \%DLCO (\%) & 87.0 & 85.1 & 88.4 \\
\hline Pulmonary hypertension (\%) & 28.1 & 27.0 & 29.0 \\
\hline Esophagus (\%) & 20.6 & 35.7 & 10.0 \\
\hline Heart $(\%)$ & 29.4 & 28.6 & 30.0 \\
\hline Kidney (\%) & 0.0 & 0.0 & 0.0 \\
\hline Joint (\%) & 57.9 & 71.4 & 50.0 \\
\hline Thrombosis $(\%)$ & 0.0 & 0.0 & 0.0 \\
\hline \multicolumn{4}{|l|}{ ANA specificity } \\
\hline Anti-topo I (\%) & 27.8 & 21.4 & 31.8 \\
\hline (levels, IU/mL) & 36.7 & 33.8 & 39.6 \\
\hline Anti-centromere $(\%)$ & 38.9 & 50.0 & 31.8 \\
\hline (levels, IU/mL) & 74.5 & 73.0 & 76.1 \\
\hline Anti-U1 RNP (\%) & 16.7 & 21.4 & 13.6 \\
\hline (levels, IU/mL) & 10.4 & 12.9 & 7.9 \\
\hline Anti-SS-A (\%) & 30.6 & 57.1 & 13.6 \\
\hline (levels, IU/mL) & 28.0 & 22.6 & 32.3 \\
\hline
\end{tabular}

The cut-off vale was set at the mean $+2 \mathrm{SD}$ of the value in healthy control subjects. m-TSS, modified Rodnan's total skin thickness score; SF, sublingual frenulum; VC, vital capacity; DLco, diffusion capacity for carbon monooxidase; ANA, antinuclear antibodies; Anti-topo I, antitopoisomerase I antibody; Anti-centromere, anti-centromere antibodies; Anti-U1 RNP, anti-U1 RNP antibodies; Anti-SS-A, anti-SS-A antibodies. ${ }^{*} p<0.05$ versus patients with normal Flt-3 ligand levels using Fisher's exact probability test.

\subsection{Correlation of serum Flt-3 ligand levels with clinical manifestations and laboratory data in patients with DM or SSc}

Then we examined correlation of serum Flt-3 ligand levels with clinical and serological features of patients with DM or SSc. As shown in Table 1, when Flt-3 ligand levels $>$ mean $+4 \mathrm{SD}$ of the values in normal subjects $(178.5 \mathrm{pg} / \mathrm{mL})$ were regarded as elevated, DM patients with elevated Flt-3 ligand levels tended to be accompanied with heliotrope rash at a significantly higher prevalence than those with normal levels $(100.0 \%$ vs. $50.0 \%, p=0.029)$. Furthermore, C-reactive protein (CRP) levels (normal values; $<0.3 \mathrm{mg} / \mathrm{dL}$ ) in DM patients with elevated Flt-3 ligand levels were significantly higher than in those without (1.48 vs. 0.19 $\mathrm{mg} / \mathrm{dL}, p=0.019)$. We could not find any significant differences between patients with and without elevated Flt-3 ligand levels in terms of the frequency of Gottron's sign, the levels of muscle enzymes, or the positive rates and the levels of antinuclear antibody (ANA) and Jo-1 antibody. Duration of disease (between the onset of symptoms and the first hospital visit) was slightly shorter in patients with elevated Flt-3 ligand levels, but not statistically significant.

On the other hand, when SSc patients were divided into two groups according to the cut-off value (136.4 $\mathrm{pg} / \mathrm{dL}$, mean $+2 \mathrm{SD}$ of normal subjects), patients with elevated Flt-3 ligand levels showed a significantly reduced prevalence of NFB as compared to those with normal levels (26.7 vs. 63.6\%, $p=0.027$, Table 2). There were no significant differences between patients with elevated Flt-3 ligand levels and those with normal levels in the frequency of major organ involvements 
or other vascular involvements including Raynaud's phenomenon, thrombosis, pitting scar or telangiectasia. Furthermore, CRP levels were not significantly different between the two groups. The positive rates and the levels of antibodies against topoisomerase I, centromere, U1 RNP or SS-A were not significantly different between patients with and without elevated Flt-3 ligand levels. Duration of disease was shorter in patients with elevated Flt-3 ligand levels, albeit insignificant.

\section{Discussion}

We demonstrated several novel findings in this study. First, levels of serum Flt-3 ligand were significantly elevated in patients with DM, SSc, SSD, and MCTD compared to normal subjects. Second, SSc patients with elevated Flt-3 ligand levels showed significantly reduced prevalence of NFB compared to those with normal levels. And lastly, in DM patients, those with elevated Flt-3 ligand levels were accompanied with significantly increased CRP levels and increased frequency of heliotrope rash than those with normal levels.

NFB is one of the earliest vascular events in SSc, and the main factor of the diagnostic criteria for SSD. Although we also performed capillaroscopy on several SSc patients with or without increased serum Flt-3 ligand levels, we could not find any significant differences in the frequency of capillaroscopic findings including bleeding, dilated loops and giant loops between the two groups, probably because the number of patients determined was limited and insufficient to allow statistically based conclusions. Considering the significant elevation of Flt-3 ligand levels in SSD patients and the reduced frequency of NFB in SSc patients with elevated Flt-3 ligand levels, there is a possibility that Flt-3 ligand may start to elevate at the prodromal stage of SSc, and have suppressive effect on NFB. Increased Flt-3 ligand levels may activate Flt-3 signal, and may stimulate vascular formation to repair vascular abnormalities of NFB in these patients.

Although patients in this study were randomly chosen, DM patients showed high CK levels $(2,400.8$ IU/L) and high CRP levels $(0.83 \mathrm{mg} / \mathrm{dL})$ on average. Thus, one of the reasons why DM patients showed the elevated serum Flt-3 ligand values is possibly due to their high disease activities at the sampling point. Higher CRP levels and a higher frequency of heliotrope rash in DM patients with elevated Flt-3 ligand levels indicated that an abnormal increase of Flt-3 ligand may be correlated with the disease activity and the formation of the skin lesions. Crowson et al. previously reported that skin lesions of DM showed more severe endothelial injury, telangiectasia, and deposition of fibrin or C5b-9 on vessels than those of lupus erythematosus, whereas the density of superficial vascular plexus was lower in lesions of DM compared to lupus erythematosus (24). Several investigators also have demonstrated a marked reduction of capillary number in the involved muscles of DM $(25,26)$. These results indicate that vascular abnormalities are present in DM, and play a role in the pathogenesis of skin lesions. Given that Flt-3 signals may be mediated in the vessel formation process, and that uncontrolled activation of angiogenic factors rather than their inactivation is thought to cause vascular abnormalities in rheumatic diseases $(27,28)$. The excessive expression of Flt-3 ligands in DM may also induce dysregulation of the vessel formation, resulting in the formation of heliotrope rash. Consistently, MCTD patients included in this study were accompanied with heliotrope rash, and showed the highest Flt-3 ligand levels among rheumatic diseases, although we could not determine ROC curve analysis or the association with clinical/laboratory findings due to the small number of the patients. On the other hand, although SLE patients are sometimes accompanied with a variety of microangiopathy phenotypes, weaker changes of vessels in SLE than in DM as described above may explain the lower serum Flt-3 ligand levels in SLE.

Taken together, our results suggested that Flt3 signal may play a different role in each rheumatic disease. Telangiectasia and endothelial injury are commonly observed both in skin lesions of DM including heliotrope rash and in the microangiopathy such as NFB $(24,29)$, which may be associated with increased Flt-3 ligand levels in patients with DM or SSc. However, there are also several differences between these two abnormalities (e.g. distribution or the presence of bleeding). Further researches on the similarities and the dissimilarities are needed in the future.

On the other hand, we had expected that the association of serum Flt-3 ligand levels and immunodysfunction in patients with DM or SSc, but the positive rates and the levels of ANA or other antibodies were not significantly different between patients with and without elevated serum Flt-3 ligand levels.

As the limitation of this study, because the onset ages of SSc, DM, SLE, and MCTD were usually different $(30,31)$, the patients were not age-matched. There was no correlation between serum Flt-3 ligand levels and the age of individuals included in this study $(r=0.399)$. Furthermore, Kinn et al. have reported that the expression levels of Flt-3 ligand in healthy skin did not show any significant correlation with aging (7), and Metcalf et al. have suggested that Flt-3 ligand levels in peripheral blood mononuclear cells were similar between adult people and old people (32). Thus, the direct correlation between aging and serum Flt-3 ligand levels is not obvious. However, the mean ages of both SSc and DM patients were higher than that of SLE patients, and the levels of Flt-3 ligand in SSc and DM 
were higher than those in SLE. Additionally, the mean age of DM or SSc patients with elevated Flt-3 ligand levels tended to be older than the counterpart. Because normal aging is usually associated with minimal/ chronic inflammation coined by inflammaging, there is a possibility that the difference of Flt-3 ligand levels can be explained by aging factor. Future studies to measure circulating levels of Flt-3 or Flt-3 ligand in normal aging as well as malignancy or viral infection are needed. On the other hand, albeit insignificant, disease duration of the elevated Flt-3 ligand groups in both SSc and DM patients was shorter than the counterpart. This result suggests that these patients may have more severe symptoms. These points should also be clarified in the future by larger studies.

In conclusion, serum Flt-3 ligand levels may be a marker of cutaneous manifestations in DM patients and a marker of microangiopathy in SSc patients. Clarifying the role of Flt-3 ligand in rheumatic disease by systematic approach is required for further understanding of the diseases and new therapeutic approaches.

\section{Acknowledgements}

This study was supported in part by a grant for scientific research from the Japanese Ministry of Education, Science, Sports and Culture, by project research on intractable diseases from the Japanese Ministry of Health, Labour and Welfare, and by Lydia O'leary Memorial Foundation.

\section{References}

1. Lyons R, Narain S, Nichols C, Satoh M, Reeves WH. Effective use of autoantibody tests in the diagnosis of systemic autoimmune disease. Ann N Y Acad Sci. 2005; 1050:217-228.

2. Szekanecz Z, Koch AE. Vascular involvement in rheumatic diseases: 'vascular rheumatology'. Arthritis Res Ther. 2008; 10:224.

3. Mulligan-Kehoe MJ, Simons M. Vascular disease in scleroderma: Angiogenesis and vascular repair. Rheum Dis Clin North Am. 2008; 34:73-79.

4. Wilhelm SM, Carter C, Tang L, et al. BAY 43-9006 exhibits broad spectrum oral antitumor activity and targets the RAF/MEK/ERK pathway and receptor tyrosine kinases involved in tumor progression and angiogenesis. Cancer Res. 2004; 64:7099-7109.

5. Li LX, Goetz CA, Katerndahl CD, Sakaguchi N, Farrar MA. A Flt3- and Ras-dependent pathway primes B cell development by inducing a state of IL-7 responsiveness. J Immunol. 2010; 184:1728-1736.

6. Smith JR, Thackray AM, Bujdoso R. Reduced herpes simplex virus type I latency in Flt-3 ligand-treated mice is associated with enhanced numbers of natural killer and dendritic cells. Immunology 2001; 102:352-358.

7. Kinn PM, Holdren GO, Westermeyer BA, Abuissa M, Fischer CL, Fairley JA, Brogden KA, Brogden NK. Age-dependent variation in cytokines, chemokines, and biologic analytes rinsed from the surface of healthy human skin. Sci Rep. 2015; 5:e10472.

8. Nakao M, Yokota S, Iwai T, Horiike S, Kashima K, Sonoda Y, Fujimoto T, Misawa S. Internal tandem duplication of the flt 3 gene found in acute myeloid leukemia. Leukemia. 1996; 10:1911-1918.

9. Stirewalt DL, Radich JP. The role of FLT3 in haematopoietic malignancies. Nat Rev Cancer. 2003; 3:650-665.

10. Tobón GJ, Renaudineau Y, Hillion S, Cornec D, Devauchelle-Pensec V, Youinou P, Pers JO. The Fms-like tyrosine kinase 3 ligand, a mediator of B cell survival, is also a marker of lymphoma in primary Sjögren's syndrome. Arthritis Rheum. 2010; 62:3447-3456.

11. Ramos MI, Perez SG, Aarrass S, Helder B, Broekstra P, Gerlag DM, Reedquist KA, Tak PP, Lebre MC. FMSrelated tyrosine kinase 3 ligand (F1t3L)/CD135 axis in rheumatoid arthritis. Arthritis Res Ther. 2013; 15:R209.

12. Subcommittee for scleroderma criteria of the American rheumatism association diagnostic and therapeutic criteria committee. Preliminary criteria for the classification of systemic sclerosis (scleroderma). Arthritis Rheum. 1980; 23:581-590.

13. Hochberg MC. Updating the American College of Rheumatology revised criteria for the classification of systemic lupus erythematosus. Arthritis Rheum. 1997; 40:1725.

14. LeRoy EC, Black C, Fleischmajer R, Jablonska S, Krieg T, Medsger TA, Rowell N, Wollheim F. Scleroderma (systemic sclerosis): Classification, subsets and pathogenesis. J Rheumatol. 1988; 15:202-205.

15. Maricq HR, Weinrich MC, Keil JE, Smith EA, Harper FE, Nussbaum AI, Leroy EC, McGregor AR, Diat F, Rosal EJ. Prevalence of scleroderma spectrum disorders in the general population of South Carolina. Arthritis Rheum. 1989; 32:998-1006.

16. Maricq HR, Mcgregor AR, Diat F, Smith EA, Maxwell DB, LeRoy EC, Weinrich MC. Major clinical diagnoses found among patients with Raynaud phenomenon from the general population. J Rheumatol. 1990; 17:11711176.

17. Ihn H, Sato S, Tamaki T, Soma Y, Tsuchida, Ishibashi $Y$, Takehara K. Clinical evaluation of scleroderma spectrum disorders using a points system. Arch Dermatol Res. 1992; 284:391-395.

18. Bohan A, Peter JB. Polymyositis and dermatomyositis (first of two parts). N Engl J Med. 1975; 292:344-347.

19. Bohan A, Peter JB. Polymyositis and dermatomyositis (second of two parts). N Engl J Med. 1975; 292:403-407.

20. Sontheimer RD. Cutaneous features of classic dermatomyositis and amyopathic dermatomyositis. Curr Opin Rheumatol. 1999; 11:475-482.

21. Alarcón-Segovia, D, Delezé M, Oria, CV, SanchezGuerrero J, Gómez-Pacheco L, Cabiedes J, Fernández L, Ponce de León S. Antiphospholipid antibodies and the antiphospholipid syndrome in systemic lupus erythematosus a prospective analysis of 500 consecutive patients. Medicine. 1989; 68:353-365.

22. Ihn H, Yamane K, Yazawa N, Kubo M, Fujimoto M, Sato S, Kikuchi K, Tamaki K. Distribution and antigen specificity of anti-U1RNP antibodies in patients with systemic sclerosis. Clin Exp Immunol. 1999; 117:383387.

23. Papadaki HA, Damianaki A, Pontikoglou C, Pyrovolaki K, Eliopoulos DG, Stavroulaki E, Eliopoulos GD. 
Increased levels of soluble flt-3 ligand in serum and long-term bone marrow culture supernatants in patients with chronic idiopathic neutropenia. Br J Haematol. 2006; 132:637-639.

24. Crowson AN, Maqro CM. The role of microvascular injury in the pathogenesis of cutaneous lesions of dermatomyositis. Hum Pathol. 1996; 27:15-19.

25. Dalakas MC. Review: An update on inflammatory and autoimmune myopathies. Neuropathol Appl Neurobiol. 2011; 37:226-242.

26. Mammen AL. Dermatomyositis and polymyositis: Clinical presentation, autoantibodies, and pathogenesis. Ann NY Acad Sci. 2010; 1184:134-153.

27. Distler O, Distler JHW, Scheid A, Acker T, Hirth A, Rethage J, Michel BA, Gay RE, Müller-Ladner U, Matucci-Cerinic M, Plate KH, Gassmann M, Gay $\mathrm{S}$. Uncontrolled expression of vascular endothelial growth factor and its receptors leads to insufficient skin angiogenesis in patients with systemic sclerosis. Circ Res. 2004; 95:109-116.

28. Jinnin M, Makino T, Kajihara I, Honda N, Makino K, Ogata A, Ihn H. Serum levels of soluble vascular endothelial growth factor receptor-2 in patients with systemic sclerosis. Br J Dermatol. 2010; 162:751-758.
29. Walker JG, Stirling J, Beroukas D, Dharmapatni K, Haynes DR, Smith MD, Ahern MJ, Roberts-Thomson PJ. Histopathological and ultrastructural features of dermal telangiectasias in systemic sclerosis. Pathology. 2005; 37:220-225.

30. Ohta A, Nagai M, Nishina M, Tomimitsu H, Kohsaka $\mathrm{H}$. Age at onset and gender distribution of systemic lupus erythematosus, polymyositis/dermatomyositis, and systemic sclerosis in Japan. 2013; 23:759-764.

31. Maldonado ME, Perez M, Pignac-Kobinger J, Marx ET, Tozman EM, Greidinger EL, Hoffman RW. Clinical and immunologic manifestations of mixed connective tissue disease in a Miami population compared to a Midwestern US caucasian population. J Rheumatol. 2008; 35:429-437.

32. Metcalf TU, Cubas RA, Ghneim K, Cartwright MJ, Grevenynghe JV, Richner JM, Olagnier DP, Wilkinson PA, Cameron MJ, Park BS, Hiscott JB, Diamond MS, Wertheimer AM, Nikolich-Zugich J, Haddad EK. Global analyses revealed age-related alterations in innate immune responses after stimulation of pathogen recognition receptors. Aging Cell. 2015; 14:421-432.

(Received September 17, 2015; Revised October 21, 2015; Accepted October 24, 2015) 